\title{
CrystEngComm
}

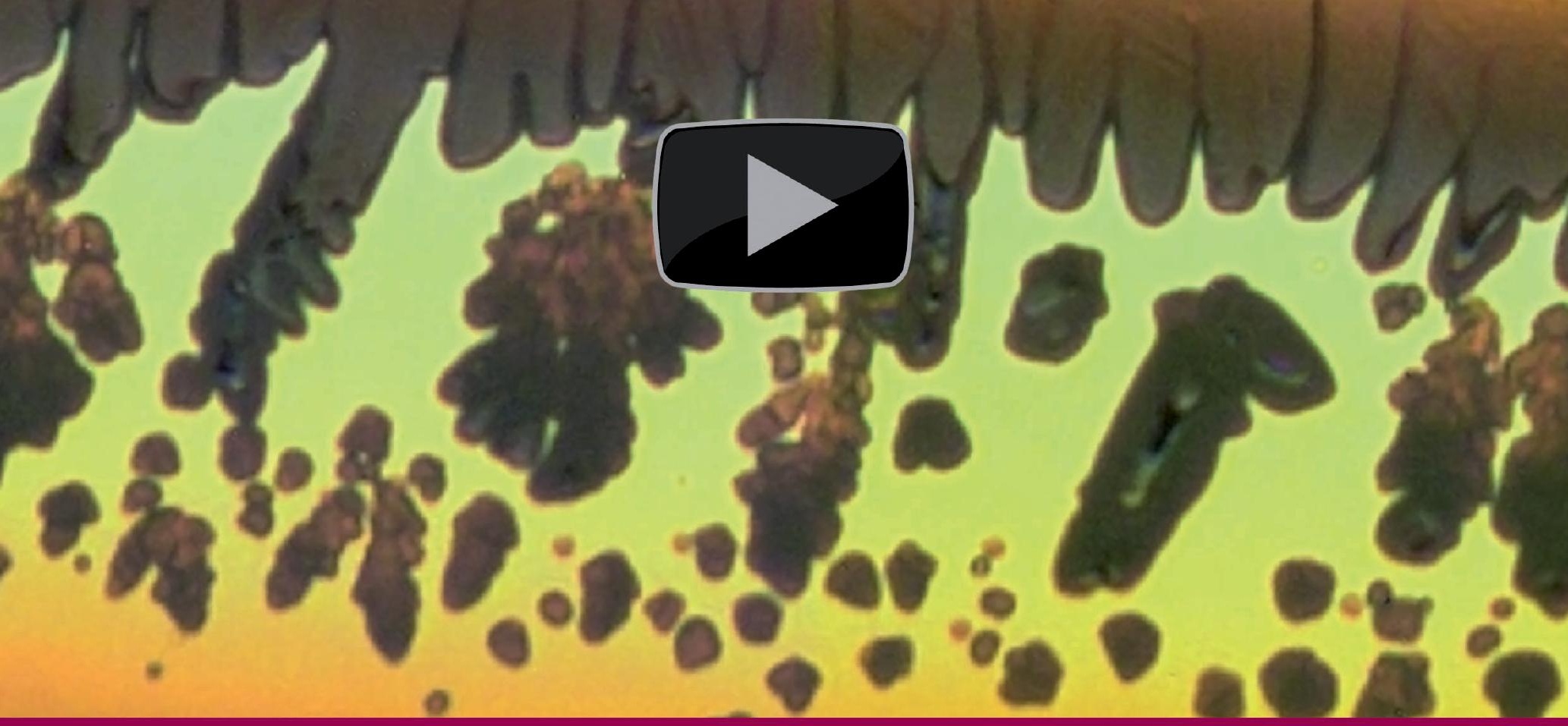

Nanocrystal growth via oriented attachment

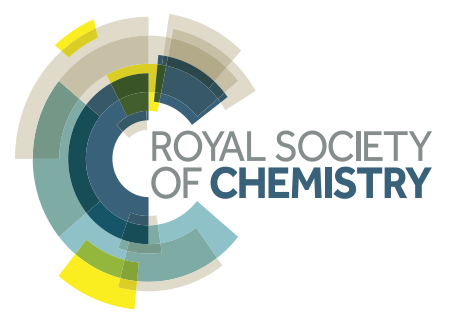




\title{
CrystEngComm
}

Cite this: CrystEngComm, 2014, 16 1443

Received 16th September 2013, Accepted 30th September 2013

DOI: $10.1039 / c 3 c e 41871 e$

www.rsc.org/crystengcomm

\section{Dynamic growth modes of ordered arrays and mesocrystals during drop-casting of iron oxide nanocubes $\uparrow$}

\author{
Michael Agthe, ${ }^{a}$ Erik Wetterskog, ${ }^{a}$ Johanne Mouzon, ${ }^{b}$ German Salazar-Alvarez ${ }^{a}$ \\ and Lennart Bergström*a
}

\begin{abstract}
The growth modes of self-assembled mesocrystals and ordered arrays from dispersions of iron oxide nanocubes with a mean edge length of $9.6 \mathrm{~nm}$ during controlled solvent removal have been investigated with a combination of visible light video microscopy, atomic force microscopy and scanning electron microscopy. Mesocrystals with translational and orientational order of sizes up to $10 \mu \mathrm{m}$ are formed spontaneously during the final, diffusion-controlled, drop-casting stage when the liquid film is very thin and the particle concentration is high. Convection-driven deposition of ordered nanocube arrays at the edge of the drying droplet is a manifestation of the so called coffee-ring effect. Dendritic growth or fingering of rapidly growing arrays of ordered nanocubes could also be observed in a transition regime as the growth front moves from the initial three-phase contact line towards the centre of the original droplet.
\end{abstract}

\section{Introduction}

Assembly of nanoparticles with a well-defined size and shape into structures with a long-range translational order can be performed with a wide range of techniques. ${ }^{1-6}$ Evaporationinduced self-assembly is one of the most simple and widely used routes to produce ordered arrays of a variety of nanoparticles and larger colloids. ${ }^{7-11}$ Drop-casting, as one of several evaporation-induced self-assembly techniques, is usually performed by distributing a dilute suspension, e.g. as a small droplet or thin film, over a solid or liquid surface followed by controlled evaporation of the carrier solvent. Although the process is simple to perform, previous work on dispersions of primarily larger monodisperse spheres has demonstrated the influence of space-filling considerations on evaporationinduced self-assembly. Together with the interactions among particles and between the particles and the substrate, evaporation-induced convection can have a dominating effect on the structure and morphology of the deposited arrays. ${ }^{12-19}$ Drying of suspensions is applied to a variety of technical products and biomedical applications where techniques such as spray drying and ink-jet printing are widely used. ${ }^{20-25}$

\footnotetext{
${ }^{a}$ Department of Materials and Environmental Chemistry, Arrhenius Laboratory, Stockholm University, SE-10691 Stockholm, Sweden.

E-mail: lennart.bergstrom@mmk.su.se; Fax: +46 8 152187; Tel: +46 8162368

${ }^{b}$ Division of Sustainable Process Engineering, Luleå University of Technology,

SE-97187 Luleå, Sweden

$\dagger$ Electronic supplementary information (ESI) available: Additional figures and

a movie. See DOI: $10.1039 / \mathrm{c} 3 c e 41871 \mathrm{e}$
}

While much of the early work on evaporation-induced self-assembly concerned the packing of monodisperse spherical silica, PMMA and polystyrene colloids into arrays that displayed a long-range mesoscopic order but no crystallographic register at the atomic scale, ${ }^{13,26-30}$ recent advances in producing non-spherical nanoparticles with a well-defined size and shape have made it possible to produce ordered arrays that are controlled by constraints given by the shape and the anisotropic particle interactions. ${ }^{1,31-33}$ Indeed, it has recently been shown that anisotropic crystalline nanoparticles are able to assemble and mutually align into periodic arrangements of crystallographic uniformity, i.e. forming an array or superlattice characterized by a high degree of directional and orientational order, ${ }^{34-39}$ which agrees with the definition of the so called mesocrystals and oriented attachment. ${ }^{40-43}$ Interestingly, studies on the oriented attachment of crystalline nanoparticles have shown that assemblies or aggregates with a high degree of crystallographic uniformity can also form under conditions where the crystal planes actually fuse together. ${ }^{42,44-46}$ The ability of anisotropic nanoparticles to self-assemble or undergo oriented attachment into larger structures with a well-defined crystallographic orientation has the potential to design new materials with anisotropic magnetic, dielectric and mechanical properties.

Visualising the self-assembly process for very small nanoparticles is challenging. The crystallization kinetics of colloidal assembly, in which the colloids are smaller than the diffraction limit of visible light, i.e. less than $200 \mathrm{~nm}$, have been studied extensively by in situ GISAXS (Grazing Incidence 
Small Angle X-Ray Scattering) ${ }^{10,31,47,48}$ and, to a lesser extent, by in situ ETEM (Environmental Transmission Electron Microscopy). ${ }^{49,50}$ However, these techniques are restricted to either indirectly measuring the kinetics by probing the structural order of the assembly (GISAXS) or are limited to small scale investigations in an environment that is strongly affected by the electron beam (ETEM).

This study focuses primarily on identifying the different dynamic growth modes during solvent evaporation of a dropcast dispersion and the type of mesostructures that are generated. We have used visible light video microscopy to determine the time-dependent growth and the morphology of the arrays and characterized the structures of the formed arrays by scanning electron microscopy and atomic force microscopy. We have identified three distinct growth regimes: diffusion-controlled assembly in a thin liquid film with high and relatively uniform particle concentration, convectiondriven assembly in the vicinity of the three-phase contact line, i.e. the so called coffee-ring stain effect, ${ }^{51}$ and a transition regime characterized by dendritic growth or so called fingering.

\section{Experimental}

Truncated iron oxide nanocubes with a narrow size distribution of $9.6 \mathrm{~nm} \pm 0.4 \mathrm{~nm}$ were obtained by thermal decomposition of an iron(III) oleate. Oleic acid (Tokyo Chemical Industry, 99\%), sodium oleate (Tokyo Chemical Industry, 97\%) and iron(III) chloride hexahydrate (Sigma-Aldrich, 97\%) were purchased and used as received. The iron(III) oleate precursor was prepared according to previously reported methods and used within 24 h. ${ }^{52,53}$ Iron(III) oleate $(10 \mathrm{mmol})$ was dissolved in a $50 \mathrm{~mL}$ solvent mixture of 1-hexadecene1-octadecene $(0.2 / 0.8$ by weight $)$ together with sodium oleate $(1.43 \mathrm{mmol})$ and oleic acid $(1.43 \mathrm{mmol})$. The solution was degassed under vacuum at $100{ }^{\circ} \mathrm{C}$ for $30 \mathrm{~min}$ under vigorous magnetic stirring. The precursor complex solution was slowly $\left(3.0{ }^{\circ} \mathrm{C} \mathrm{min}^{-1}\right)$ heated to $\mathrm{ca} .315^{\circ} \mathrm{C}$ under vigorous magnetic stirring, refluxed for $30 \mathrm{~min}$ and then rapidly cooled to room temperature. The mother liquor was extracted five times with ethanol by decantation. A small amount of toluene (1-2 mL) was added to disperse the particles after each decantation step. The extraction and subsequent drying in vacuum yielded a viscous paste with an iron oxide content estimated to be $44 \mathrm{wt} . \%$ by thermogravimetric analysis at $800{ }^{\circ} \mathrm{C}$ in synthetic air using a Perkin Elmer TGA-7 (Perkin Elmer, USA). The dispersion for the drop-casting studies was prepared by diluting and redispersing the paste in toluene together with $50 \mu \mathrm{g}$ oleic acid per $1 \mathrm{mg}$ of iron oxide to yield a stock dispersion with a concentration of $8.7 \mathrm{mg}$ iron oxide per $\mathrm{mL}$ toluene. Possible agglomerates were removed by centrifuging the stock dispersion for $10 \mathrm{~min}$ at $18000 \mathrm{RPM}$ and the final dispersion was diluted $1: 2$ with toluene.

HR-TEM images for nanoparticle characterization were acquired using a JEOL JEM-2100 microscope (JEOL, Japan, $\mathrm{LaB}_{6}$ filament at $200 \mathrm{kV}, f=2.7 \mathrm{~mm}, C_{\mathrm{s}}=1.4 \mathrm{~mm}, C_{\mathrm{c}}=1.8 \mathrm{~mm}$, point-resolution $=2.5 \AA$, lattice-resolution $=1.4 \AA$ ) equipped with a Gatan SC1000 ORIUS camera. Nanocube size distribution was determined by manually measuring 240 nanocube edge lengths in the HR-TEM images. Image magnification was calibrated using the known lattice spacing, e.g. $d_{220}$ or $d_{400}$, in the inverse spinel structure of $\mathrm{Fe}_{3} \mathrm{O}_{4}$ (see Fig. S2 in the ESI $\dagger$ ).

Drop-casting of the dilute nanocube dispersions was performed on flat $\mathrm{SiO}_{2}$ substrates in chambers where the evaporation rate could be controlled, using e.g. an additional toluene reservoir to maintain a high partial pressure. The timeresolved study experiment took place in a custom-made, allTeflon chamber design featuring detachable gas inlet and outlet tubing (inner tube diameter of $1 \mathrm{~mm}$ ), a $c a$. $300 \mu \mathrm{L}$ large solvent reservoir to prolong evaporation, and a Kalrez rubber sealing. The chamber has a window (no. $0,24 \times 60 \mathrm{~mm}^{2}$, Menzel Deckgläser, Germany) for microscopy studies with long working distance objectives. A Peltier element beneath the substrate automatically controlled the temperature to a set value of $20{ }^{\circ} \mathrm{C}$, and a rotameter (FT-042-15-SA-VA, Aalborg Instruments \& Controls, Inc., USA) was used to manually control the inlet flow of 3 sccm dry nitrogen gas.

A flat quartz disc (QSX-303, Q-Sense AB, Sweden) coated with $\mathrm{a} \mathrm{SiO}_{2}$ surface layer (RMS surface roughness of $3 \mathrm{~nm}$ ) was used as a substrate for the time-resolved study. The substrate was cleaned by rinsing in ultrapure Milli-Q water and ethanol (99.9\%, VWR International) followed by blow-drying in a nitrogen gas flow. Immediately afterwards, the substrate was placed into a UV/ozone cleaning chamber (UV/Ozone ProCleaner, BioForce Nanosciences, USA) for $20 \mathrm{~min}$ to remove any remaining organic residue. The substrate's surface was functionalized by placing the substrate and $20 \mu \mathrm{L}$ of perfluorinated silane PFOTES $(1 \mathrm{H}, 1 \mathrm{H}, 2 \mathrm{H}, 2 \mathrm{H}$-perfluorooctyltriethoxysilane, $98 \%$, Sigma-Aldrich) into a sealed glass bottle. Silanization was accelerated by heat treatment for $3 \mathrm{~h}$ at $120^{\circ} \mathrm{C}$ and for an additional $1.5 \mathrm{~h}$ at $140{ }^{\circ} \mathrm{C}$. The silanized surface on a welldefined annular section of the substrate was removed by placing a custom made Teflon ring $(14 \mathrm{~mm}$ outer diameter and $9 \mathrm{~mm}$ inner diameter) on the sensor and irradiating the exposed area for $30 \mathrm{~min}$ under UV light. This treatment creates a clean, circular, hydrophilic surface in the centre and an omniphobic surface elsewhere on the substrate.

Video microscopy in reflection mode was performed on a Nikon Eclipse FN1 light microscope (Nikon, Japan) equipped with a $10 \times$ eyepiece and a $50 \times$ long working distance objective (WD $17 \mathrm{~mm}$, NA 0.45). Attached to the microscope is an inline camera with a 2 megapixel CCD sensor (Kappa Zelos-02150C GV, Kappa Optronics GmbH, Germany). All adjustable camera parameters were set manually for best performance, and images were saved approximately every 3 seconds in uncompressed TIFF format.

Scanning electron microscopy (SEM) was carried out on a JEOL JSM-7000F microscope (JEOL, Japan), equipped with a Schottky-type FEG and operating at an accelerating voltage of $15 \mathrm{kV}$ and a working distance of $6 \mathrm{~mm}$ and $10 \mathrm{~mm}$. In order to access the microscopic features of the ordered arrays, excess organics were removed by UV/ozone cleaning for up to 5 hours. The XHR-SEM (extreme high-resolution SEM) imaging 
shown in Fig. 6c was performed on a FEI Magellan 400 microscope (FEI, USA) using the through-lens detector (TLD) in immersion mode at a working distance of $2.1 \mathrm{~mm}$. The electron beam was operated at an accelerating voltage of $3 \mathrm{kV}$ and a current of $6.3 \mathrm{pA}$.

Atomic force microscopy (AFM) measurements were carried out on a Veeco BioScope II (Veeco, USA). SPM probes (NSC18/AIBS, tip radius $<10 \mathrm{~nm}, f_{\mathrm{r}}=75 \mathrm{kHz}, k=3.5 \mathrm{~N} \mathrm{~m}^{-1}$, $\mu$ masch, USA) were pre-tested on an atomically flat HOPG surface and used in tapping mode in order to avoid accumulation of organic residue on the SPM probe tip. The AFM images were processed using either the WSxM 5.0 (ref. 54) or the Gwyddion 2.32 software package. The topography image (Fig. 1b) was flattened using the "flatten plus" method in WSxM, matching the substrate area with the lowest flat regions in the image (i.e. quartz substrate).

\section{Results and discussion}

Drop-casting the dilute nanoparticle suspension onto the substrate results in the formation of particle arrays or deposits as the solvent evaporates. Before presenting the data from the time-dependent study, we will briefly describe the features of the mesocrystals formed during the final drop-casting stage when the liquid film is very thin and the particle concentration is high.

Fig. 1 shows that mesocrystals with a high degree of order form when dilute suspensions of oleic-acid-coated iron oxide nanocubes dispersed in a mixture of toluene and high-boiling additives such as oleic acid are drop-cast onto a flat substrate and allowed to slowly evaporate. It is important to clarify that the relatively large iron oxide nanocubes used in this study (average edge length of $9.6 \mathrm{~nm} \pm 0.4 \mathrm{~nm}$ ) are able to form highly ordered self-assembled arrays in the absence of any external magnetic field (see Fig. 1a). This is in contrast with our previous work on smaller nanocubes (average edge length of $8.5 \mathrm{~nm}$ ), where it was found that a weak magnetic field had to be applied during drop-casting to generate highly ordered mesocrystals. ${ }^{31,34}$ Estimates of the van der Waals interaction energy using an accurate estimate of the Hamaker constant of $18 \mathrm{zJ}$ for maghemite interacting across toluene suggest that the larger nanocubes can indeed form stable clusters also in the absence of an additional magnetic-field induced dipolar attraction. ${ }^{55}$

Light microscopy images (see the movie in the ESI $\dagger$ with corresponding playback time from 1:22 min until the end) show that the individual cuboidal arrays in fact cover most of the central region of the original droplet. The high-resolution SEM image in Fig. 1a and the sharp spots in the corresponding fast Fourier transform (FFT) clearly demonstrate that the individual nanocubes have assembled in a well-defined crystallographic register. This is also reflected in the mesocrystal growth habit, i.e. the crystal morphology. The mesocrystals in Fig. 1 are cuboidal, i.e. projected as squares, owing to their body-centered tetragonal (bct) mesostructure. The structure of the assembled mesocrystals was analyzed by GISANS (Grazing Incidence Small Angle Neutron Scattering) and they were found to crystallize as a bct 2D-powder with unit cell parameters $a=b=13.5 \mathrm{~nm}$ and $c=23.8 \mathrm{~nm}$, where the mesocrystal $c$-direction is perpendicular to the substrate surface. ${ }^{56}$

The line scan in Fig. 1d (the white line in Fig. 1b) shows that the mesocrystals have a size of several $\mu \mathrm{m}$ across with a height of $\approx 0.3-1 \mu \mathrm{m}$. Furthermore, the mesocrystal facets are well resolved in the tapping-mode phase image (Fig. 1c).

The relatively uniform height distribution and the flat top surface structure of the mesocrystals shown in Fig. 1 suggest
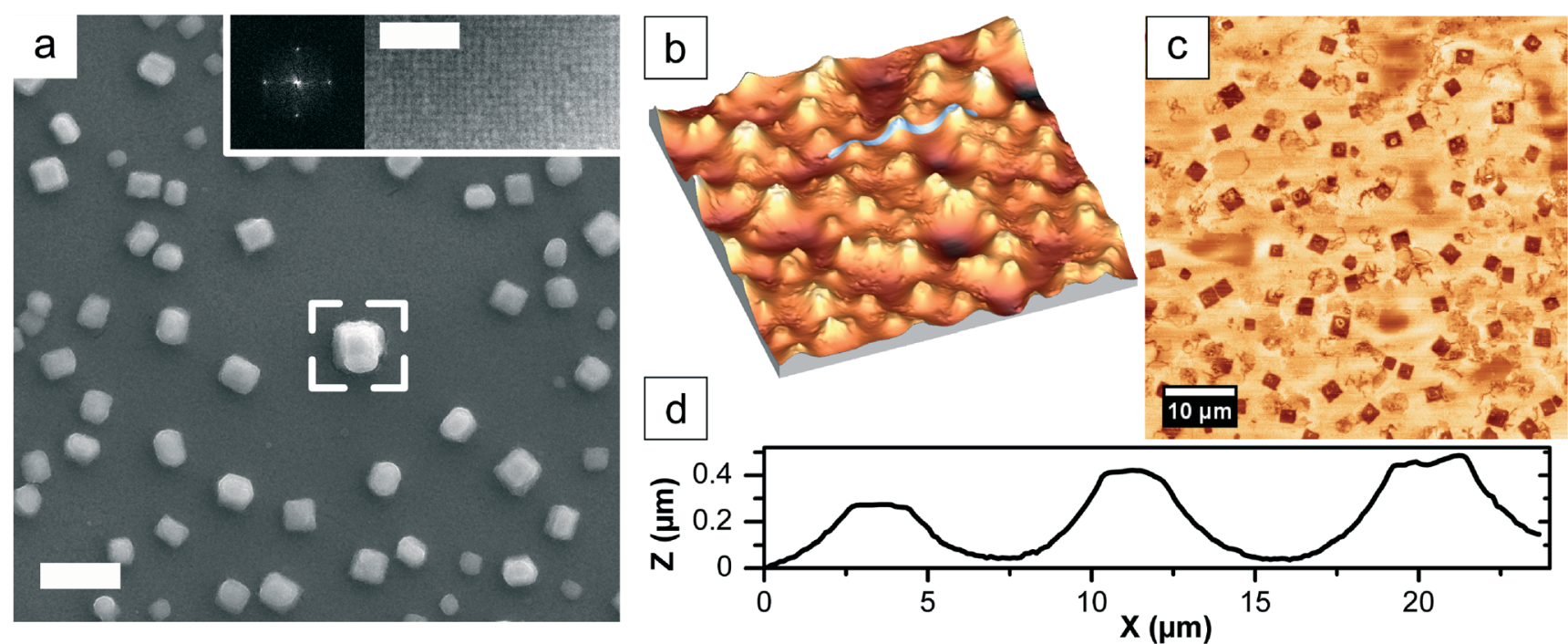

Fig. 1 SEM and AFM imaging of iron oxide mesocrystals produced by slow evaporation of a dilute dispersion of $9.6 \mathrm{~nm}$ iron oxide nanocubes. (a) The SEM image illustrates the formation of cuboidal iron oxide mesocrystals. The inset, taken from the framed area, shows a high-resolution SEM image of the top surface of the mesocrystals and its corresponding FFT pattern clearly indicates the high degree of lateral nanoparticle ordering. Scale bar, $2 \mu \mathrm{m}$ and inset, $100 \mathrm{~nm}$. (b) AFM tapping mode topology image. Scan size: $60 \mu \mathrm{m}$. Z-range: 0-940 nm. (c) AFM tapping mode phase image. (d) Height profile across the substrate, following the white line shown in (b). 
that the maximum height may be determined by the thickness of the dispersion film during the mesocrystal growth stage. Although difficult to determine with great accuracy, the film thickness at the onset of mesocrystallisation is in the order of micrometers, evident from the development of Newton rings in the dispersion film. The evaporation rate is significantly slower during this final drop-casting stage as the liquid phase is now enriched in oleic acid and high-boiling point synthesis solvent residue. ${ }^{57}$

Previous work on dispersions of anisotropic nanoparticles has primarily focussed on optimizing the drop-casting conditions for the formation of highly ordered arrays. We have previously shown that it is possible to produce ordered arrays of self-assembled iron oxide nanocube mesocrystals that display a high translational and orientational order if the evaporation is slow. ${ }^{31,34}$ However, the drop-casting process goes through several stages and we will, in this study, illustrate and discuss the formation and structure of the particle arrays that form at all stages, from the convection-driven assembly at early times to the diffusion-driven mesocrystallization.

In order to gain an insight into the different growth modes during the early and late stages of solvent evaporation, we have performed drop-casting experiments in a chamber described in Fig. 2 where the evaporation rate, fluid flow and position of the three-phase contact line are controlled or at least possible to manipulate.

The drop of the dilute dispersion of the iron oxide nanocubes spread on the hydrophilic central part of the substrate, where the edge of the drop became pinned to the edge of the outer, fluorosilane-functionalized and omniphobic region. The flow of nitrogen gas enhanced the evaporation rate and could also be used to induce a radial fluid flow into the droplet. We have recorded the evolution and formation of nanoparticle arrays in the dispersion droplet during solvent evaporation. The recording of a representative experiment can be found in the ESI. $\dagger$ It is possible to distinguish three distinct growth regimes, which may be identified as: coffeering deposition or convection-driven assembly, fingering or dendritic growth of nanoparticle assemblies and finally the diffusion-controlled mesocrystallization that was described above. These three growth regimes are separated in time and space and also display distinct differences in the growth behaviour and the morphology of the formed nanoparticle arrays (Fig. S3 in the ESI†).

In a typical drop-casting experiment, a droplet of about $15 \mu \mathrm{l}$ consisting of a dilute dispersion of oleic-acid coated iron oxide nanocubes in a toluene-based solvent was placed on the non-functionalized region of the $\mathrm{SiO}_{2}$ substrate where droplet spreading was controlled by the omniphobic region (see Fig. 2b). The droplet was allowed to evaporate at a temperature of $20^{\circ} \mathrm{C}$ and the formation of nanoparticle arrays was continuously monitored by video microscopy. In the experiment displayed in Fig. 3a, the first observation of a particle deposit is denoted as $t_{0}$. The formation of a ring-like particle structure that surrounds the entire perimeter of the droplet strongly resembles coffee-ring deposits from previous studies on drying pinned dispersion droplets of nanoparticles and larger colloids. ${ }^{15,51,58-60}$ The initial assembly occurred directly at the three-phase contact line and spread along the perimeter ( $p$-axis) until a complete arc was formed (see the time lapse video in the ESI $\dagger$ ). Rather than following a continuous layer-tolayer stacking sequence starting from a monolayer to a bilayer and so forth, Fig. $3 \mathrm{~b}$ and $\mathrm{S} 4 \uparrow$ suggest that a relatively thick and surprisingly well-ordered nanoparticle array is quickly formed, probably assisted by the radial flow of nitrogen gas.

During our experiment, we enhanced the evaporative flux gradient by flowing nitrogen across the droplet, thus pushing the liquid outwards and preventing toluene to establish and equilibrate the vapour concentration gradient with the surrounding atmosphere. The relatively thick coffee-ring nanoparticle arrays invariably always display cracks.

Crack formation in drying droplets is beyond the scope of this paper, but as has been summarized by Routh, the main driving force for cracking is capillary pressure and its interplay with shear stresses arising from the substrate. ${ }^{61}$ Cracks inside the nanoparticle assemblies are also a result of the stresses caused by the removal of organic substances by UV/ozone treatment prior to SEM imaging.

The convection-driven assembly results in the development of a macroscopic growth front of the "coffee-ring stain"
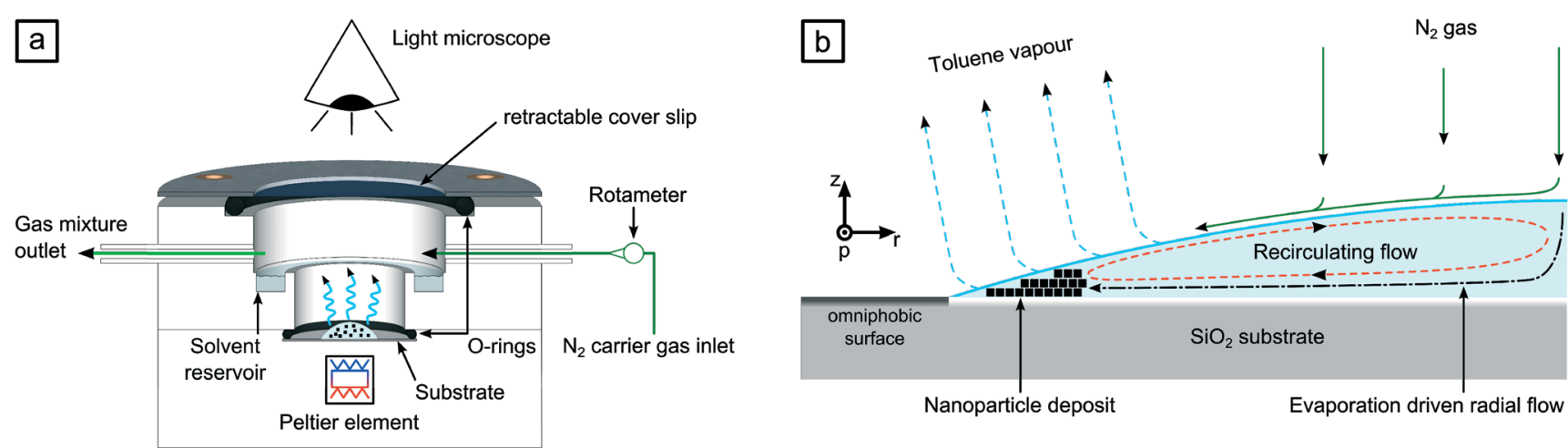

Fig. 2 Schematic illustration of the drop-casting chamber and the evaporating droplet. Light microscopy imaging of an evaporating drop consisting of oleic acid capped iron oxide nanocubes in a toluene-based fluid was carried out in an environmentally controlled chamber as shown in (a). The side view of one half of a droplet positioned on a functionalized silica substrate is shown in (b). 
that propagates towards the centre of the droplet (along the $r$ - and $z$-axes). The initially smooth growth front, which is the interface between the nanoparticle array and the dispersion, starts to split at longer evaporation times $\left(t>t_{0}+1024 \mathrm{~s}\right.$ in Fig. 3a) and eventually forms instabilities (see e.g. Fig. 3a at $t=t_{0}+1443 \mathrm{~s}$ ), which then quickly grow into fractal-like structures. Indeed, such a transition between a smooth "coffee-ring stain" and fingering has been observed in previous drying droplet studies where it was attributed to Marangoni effects caused by small temperature differences through evaporative cooling. ${ }^{62-70}$

Further support for the critical effect of small variations in chemical potential is the observation illustrated in Fig. 4a that unstable spheroidal nanoparticle assemblies or aggregates of diameters up to $3 \mu \mathrm{m}$ constantly form, coalesce with other liquid-like aggregates and dissolve again (see the movie
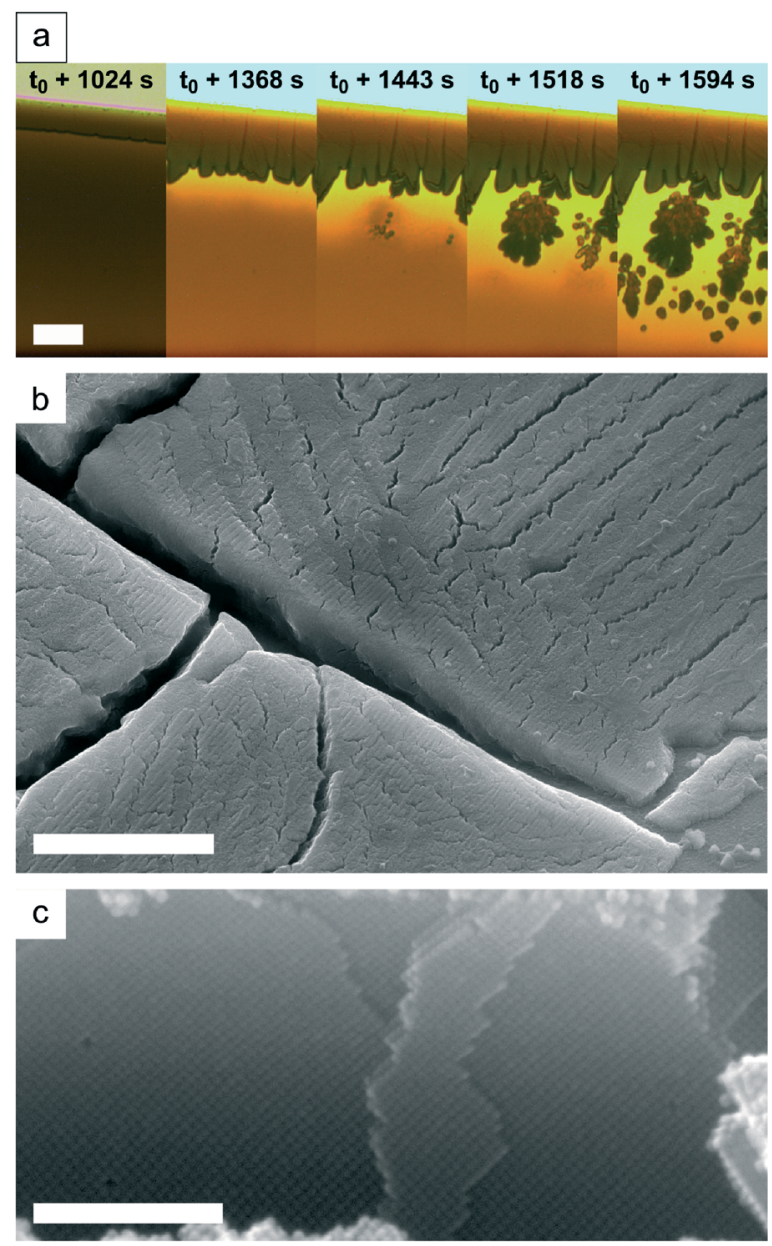

Fig. 3 Images in a time sequence illustrating convection-driven assembly and dendritic growth of nanoparticle arrays during solvent evaporation from a pinned dispersion droplet. The time at which the first coffee-ring deposit is detected at the three-phase contact line is denoted as $t_{0}$. The images in (a) show the time-dependent transition from coffee-ring deposition of a nanoparticle array with a well-defined thickness to dendritic growth. The SEM image in (b) shows the (cracked) nanoparticle deposits in the vicinity of the contact line. The highresolution SEM image in (c) illustrates the highly ordered interior of the coffee-ring deposit. Scale bar in (a) $20 \mu \mathrm{m}$, (b) $3 \mu \mathrm{m}$ and (c) $200 \mathrm{~nm}$. in the ESI $\dagger$ with corresponding playback time from 1:08 min to $1: 12 \mathrm{~min})$.

The unstable spheroidal assemblies may coalesce with the "coffee-ring" deposit (see e.g. the white frame in Fig. 4a at $t=t_{0}+1443 \mathrm{~s}$ ) and eventually solidify into "fingers" or
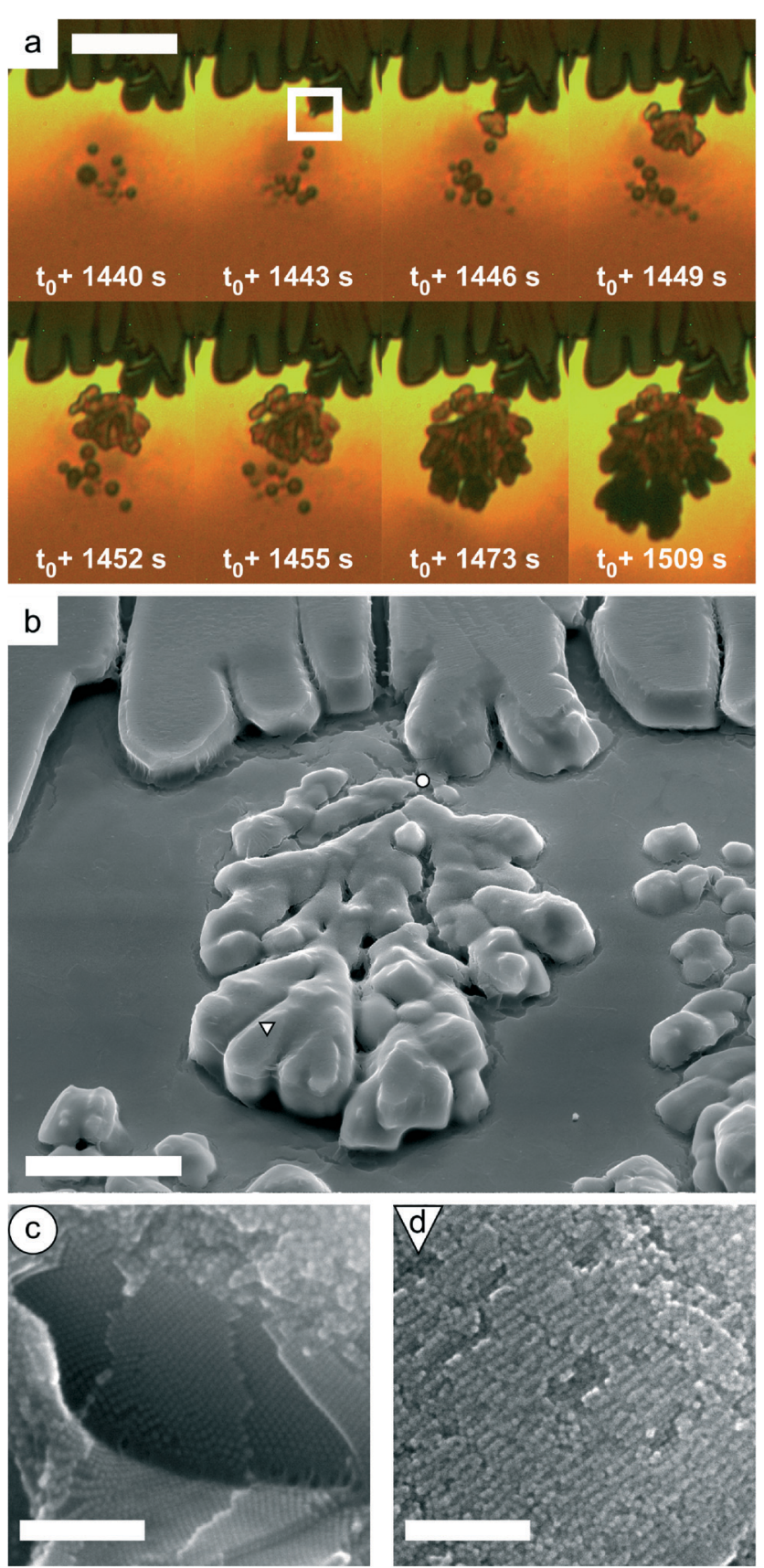

Fig. 4 Images illustrating the dendritic growth of nanoparticle arrays during drop-casting. The video microscopy images over a restricted time window shown in (a) are obtained from the same experiment shown in Fig. 3. The SEM image in (b) depicts a tilted view $\left(41.8^{\circ}\right)$ of the same fingering instability with fractal-like, dense-branches being related to high NP concentration. The high-magnification SEM image in (c) shows a fractured dendritic-like nanoparticle array illustrating the highly ordered interior and (d) shows an example of the defective surface structure. Scale bar in (a) $20 \mu \mathrm{m}$, (b) $10 \mu \mathrm{m}$, and (c) and (d) $200 \mathrm{~nm}$. 
dendritic-like arrays. It is interesting to note that the densely ramified dendritic-like structures formed fast $\left(t_{\mathrm{f}, \text { dendritic }} \approx 150 \mathrm{~s}\right)$ in comparison to the convection-driven ring-like arrays $\left(t_{\mathrm{f} \text {,coffee-ring }} \approx 1600 \mathrm{~s}\right)$. The growth quickly slows down as the surrounding liquid is depleted from the iron oxide nanocubes, eventually resulting in the termination of further growth of the dendritic-like arrays.

The nanoparticle arrays formed during the dendritic-like growth displayed in Fig. 4 are characterized by a disordered or defective surface structure (see Fig. 4d), but the interior structure shown in Fig. 4c is highly ordered.

The transport of the relatively large, unstable, liquid-like "precursor" assemblies is not only driven by diffusion but also controlled by the competition between convective motion and recirculating flow, ${ }^{63,69,70}$ i.e. "coffee-ring" convectiondriven transport and Marangoni flow-induced recirculation, which can result in "convection cell"-like flow pattern as illustrated in Fig. 5 and is formed comparatively similar to the observations by Haw et al. ${ }^{27}$

The final stage of drop-casting the iron oxide nanocube dispersions involves the formation of individual cuboidal, highly ordered particle arrays. This stage, which indeed produces mesocrystals, takes place when the dendritic-like growth is nearly terminated (see the movie in the ESI $\dagger$ with corresponding playback time from 1:14 min to the end and Fig. $\mathrm{S} 3 \dagger$ ) and occurs as discussed previously when the liquid film is thin and the particle concentration is high. Fig. 6 displays examples of the mesocrystals that form across a large fraction of the surface initially covered by the drying droplet. The iron oxide nanocubes mutually self-assemble into a bct structure that is evident from the high-resolution SEM image in Fig. 6c in which the schematic unit cell and its indicated parameters show the typical arrangement according to investigations by GISANS. ${ }^{56}$ The top surface of the mesocrystal presents the four-fold arrangement of the unit cell's symmetric basal plane with parameter $a$, and the fracture surface reveals the $c$-direction.

We have shown that drop-casting of a dilute iron oxide nanocube dispersion results in the assembly of arrays with distinct differences in morphology and growth mode. However, all of the arrays are highly ordered, suggesting that the packing constraints and particle interactions dominate over

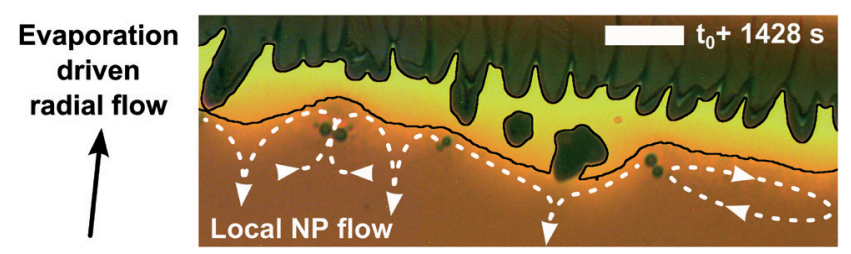

Fig. 5 Formation of a "convection cell"-like flow pattern at the heterogeneous interface between the particle array deposit and the drying fluid dispersion. Arrows indicate the flow direction from the convex, i.e. high pressure regions, towards the bulk fluid. The arrow directions and dotted lines are a guide to the eye to show local flows. The black line indicates an interface between assemblies and the liquid. Scale bar, $20 \mu \mathrm{m}$.
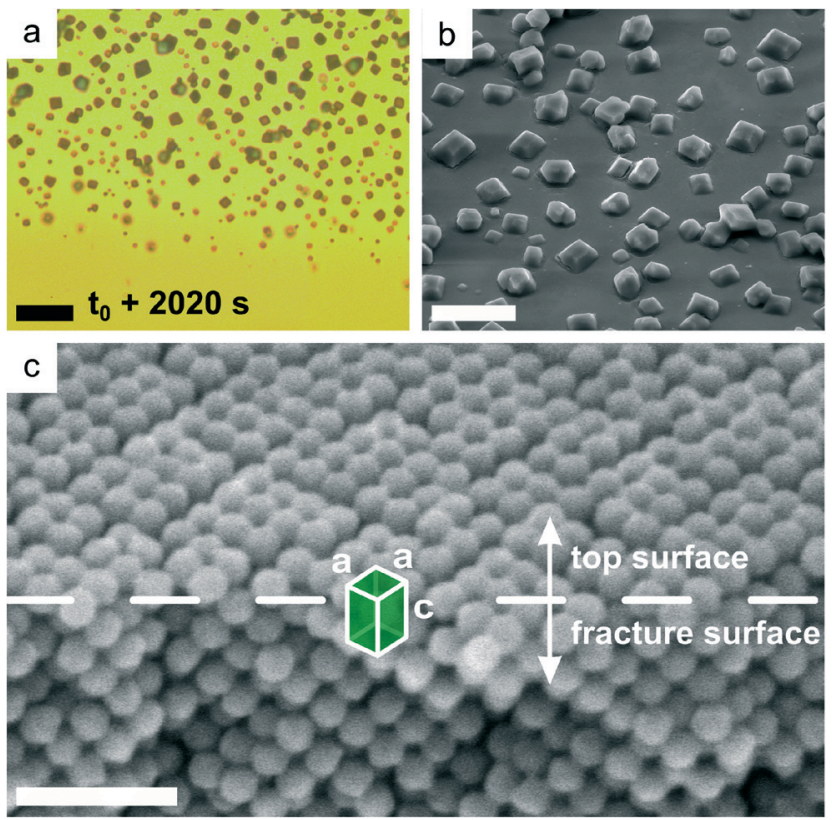

Fig. 6 Images illustrating mesocrystallization during the final stage of drop-casting iron oxide nanocube dispersions. The video microscopy snapshot in (a) was taken about $200 \mu \mathrm{m}$ inward from the pinned threephase contact line of the receding droplet. The SEM image in (b) depicts individual cuboidal mesocrystals dispersed on the substrate (sample stage was tilted by $41.8^{\circ}$ ). In (c), an XHR-SEM image of a fractured mesocrystal, tilted by $30^{\circ}$, clearly shows the bct structure (unit cell with parameters $a$ and c). Scale bar in (a) $20 \mu \mathrm{m}$, (b) $10 \mu \mathrm{m}$ and (c) $50 \mathrm{~nm}$.

the differences in growth mode and formation kinetics. While there is significant evidence that the ordered arrays formed during the final, diffusion-controlled, drop-casting stage can display both a translational and an orientational order, i.e. they are in fact mesocrystals, it is not at present possible to determine if the ordered arrays produced during the initial convection-driven deposition or the following dendritic-like growth or fingering are also mesocrystals. However, the possibility of producing mesocrystals by dropcasting surfactant-coated nanoparticles suggests that processes resembling oriented attachment can play an important role for systems that are not in direct atomic contact.

\section{Conclusions}

Summarising, we have investigated the self-assembly of iron oxide nanocubes into mesocrystals and ordered arrays during the controlled solvent evaporation in drop-casting experiments. Using visible light video microscopy, we observe that the assembly occurs in three successive stages: convectiondriven deposition of nanoparticles at the edge of the drying droplet, i.e., coffee-ring stain effect; dendritic growth (or fingering) in a transition regime; and finally spontaneous diffusion-controlled formation of mesocrystals. Scanning electron microscopy shows that the produced arrays are ordered and suggest that they are all, in fact, mesocrystals, despite the differences in morphology and growth mode. Atomic force microscopy imaging indicates that the 
formation of mesocrystals obtained at the last stage occurs when the liquid film is very thin but the nanoparticle concentration is still relatively high. Understanding of the drying phenomena during controlled solvent evaporation could lead to tuning of the three-dimensional growth of ordered nanoparticles arrays, with tailored physical properties and surface structures.

\section{Acknowledgements}

This work was financed by the Swedish Research Council (VR). The Knut and Alice Wallenberg (KAW) Foundation is acknowledged for providing the electron microscopy and atomic force microscopy facilities at Stockholm University as well as the FEI Magellan 400 XHR-SEM at Luleå University of Technology. We gratefully acknowledge Per Jansson for his input on the design and manufacturing of the experimental chamber.

\section{Notes and references}

1 K. J. M. Bishop, C. E. Wilmer, S. Soh and B. A. Grzybowski, Small, 2009, 5, 1600.

2 M. I. Bodnarchuk, M. V. Kovalenko, S. Pichler, G. Fritz-Popovski, G. Hesser and W. Heiss, ACS Nano, 2010, 4, 423.

3 J. Henzie, M. Grünwald, A. Widmer-Cooper, P. L. Geissler and P. Yang, Nat. Mater., 2011, 11, 131.

4 S. Mann, Nat. Mater., 2009, 8, 781.

5 C. B. Murray, C. R. Kagan and M. G. Bawendi, Annu. Rev. Mater. Sci., 2000, 30, 545.

6 T. Wang, J. Zhuang, J. Lynch, O. Chen, Z. Wang, X. Wang, D. LaMontagne, H. Wu and Y. C. Cao, Science, 2012, 338, 358.

7 T. P. Bigioni, X.-M. Lin, T. T. Nguyen, E. I. Corwin, T. A. Witten and H. M. Jaeger, Nat. Mater., 2006, 5, 265.

8 C. J. Brinker, Y. Lu, A. Sellinger and H. Fan, Adv. Mater., 1999, 11, 579.

9 Z. Jiang, X.-M. Lin, M. Sprung, S. Narayanan and J. Wang, Nano Lett., 2010, 10, 799.

10 S. Narayanan, J. Wang and X.-M. Lin, Phys. Rev. Lett., 2004, 93.

11 S. Wong, V. Kitaev and G. A. Ozin, J. Am. Chem. Soc., 2003, 125, 15589.

12 M. Abkarian, J. Nunes and H. A. Stone, J. Am. Chem. Soc., 2004, 126, 5978.

13 N. Denkov, O. Velev, P. Kralchevski, I. Ivanov, H. Yoshimura and K. Nagayama, Langmuir, 1992, 8, 3183.

14 R. Deegan, Phys. Rev. E: Stat. Phys., Plasmas, Fluids, Relat. Interdiscip. Top., 2000, 61, 475.

15 Á. G. Marín, H. Gelderblom, D. Lohse and J. H. Snoeijer, Phys. Rev. Lett., 2011, 107, 085502.

16 C. Nobile, L. Carbone, A. Fiore, R. Cingolani, L. Manna and R. Krahne, J. Phys.: Condens. Matter, 2009, 21, 264013.

17 E. Rabani, D. R. Reichman, P. L. Geissler and L. E. Brus, Nature, 2003, 426, 271.

18 A. F. Routh and W. B. Russel, AIChE J., 1998, 44, 2088.
19 Y. Xia, Y. Yin, Y. Lu and J. McLellan, Adv. Funct. Mater., 2003, 13, 907.

20 D. Brutin, B. Sobac, B. Loquet and J. Sampol, J. Fluid Mech., 2011, 667, 85.

21 R. Fürstner, W. Barthlott, C. Neinhuis and P. Walzel, Langmuir, 2005, 21, 956.

22 M. C. Heine and S. E. Pratsinis, Ind. Eng. Chem. Res., 2005, 44, 6222 .

23 W. Kern, J. Electrochem. Soc., 1990, 137, 1887.

24 F. C. Krebs, Sol. Energy Mater. Sol. Cells, 2009, 93, 394.

25 J. A. Lim, W. H. Lee, H. S. Lee, J. H. Lee, Y. D. Park and K. Cho, Adv. Funct. Mater., 2008, 18, 229.

26 R. Deegan, O. Bakajin, T. Dupont, G. Huber, S. Nagel and T. Witten, Phys. Rev. E: Stat. Phys., Plasmas, Fluids, Relat. Interdiscip. Top., 2000, 62, 756.

27 M. D. Haw, M. Gillie and W. C. K. Poon, Langmuir, 2002, 18, 1626.

28 K. Nagayama, Colloids Surf., A, 1996, 109, 363.

29 S. Yang, H. Míguez and G. Ozin, Adv. Funct. Mater., 2002, $12,425$.

30 M. Zacharias, J. Heitmann, R. Scholz, U. Kahler, M. Schmidt and J. Bläsing, Appl. Phys. Lett., 2002, 80, 661.

31 S. Disch, E. Wetterskog, R. P. Hermann, G. Salazar-Alvarez, P. Busch, T. Brückel, L. Bergström and S. Kamali, Nano Lett., 2011, 11, 1651.

32 K. S. Khalil, A. Sagastegui, Y. Li, M. A. Tahir, J. E. S. Socolar, B. J. Wiley and B. B. Yellen, Nat. Commun., 2012, 3, 794.

33 K. Miszta, J. de Graaf, G. Bertoni, D. Dorfs, R. Brescia, S. Marras, L. Ceseracciu, R. Cingolani, R. van Roij, M. Dijkstra and L. Manna, Nat. Mater., 2011, 10, 872.

34 A. Ahniyaz, Y. Sakamoto and L. Bergstrom, Proc. Natl. Acad. Sci. U. S. A., 2007, 104, 17570.

35 K. Bian, J. J. Choi, A. Kaushik, P. Clancy, D.-M. Smilgies and T. Hanrath, ACS Nano, 2011, 5, 2815.

36 L. Li, Y. Yang, J. Ding and J. Xue, Chem. Mater., 2010, 22, 3183.

37 P. Simon, E. Rosseeva, I. A. Baburin, L. Liebscher, S. G. Hickey, R. Cardoso-Gil, A. Eychmüller, R. Kniep and W. Carrillo-Cabrera, Angew. Chem., Int. Ed., 2012, 51, 10776.

38 J. Zhang, Z. Luo, Z. Quan, Y. Wang, A. Kumbhar, D.-M. Smilgies and J. Fang, Nano Lett., 2011, 11, 2912.

39 R. K. Zheng, H. Gu, B. Xu, K. K. Fung, X. X. Zhang and S. P. Ringer, Adv. Mater., 2006, 18, 2418.

40 H. Cölfen and M. Antonietti, Mesocrystals and Nonclassical Crystallization, John Wiley \& Sons, Ltd., Chichester, UK, 2008.

41 E. R. Leite and C. Ribeiro, in Crystallization and Growth of Colloidal Nanocrystals, ed. E. R. Leite and C. Ribeiro, Springer New York, New York, NY, 2012, p. 45.

42 R. L. Penn, Science, 1998, 281, 969.

43 R.-Q. Song and H. Cölfen, Adv. Mater., 2010, 22, 1301.

44 J. F. Banfield, Science, 2000, 289, 751.

45 Q. Zhang, S.-J. Liu and S.-H. Yu, J. Mater. Chem., 2009, 19, 191.

46 L.-S. Zhong, J.-S. Hu, H.-P. Liang, A.-M. Cao, W.-G. Song and L.-J. Wan, Adv. Mater., 2006, 18, 2426.

47 P. Siffalovic, E. Majkova, L. Chitu, M. Jergel, S. Luby, I. Capek, A. Satka, A. Timmann and S. V. Roth, Small, 2008, 4, 2222. 
48 S. Disch, E. Wetterskog, R. P. Hermann, D. Korolkov, P. Busch, P. Boesecke, O. Lyon, U. Vainio, G. Salazar-Alvarez, L. Bergström and T. Brückel, Nanoscale, 2013, 5, 3969.

49 J. Park, H. Zheng, W. C. Lee, P. L. Geissler, E. Rabani and A. P. Alivisatos, ACS Nano, 2012, 6, 2078.

50 H. Yoshida, S. Takeda, T. Uchiyama, H. Kohno and Y. Homma, Nano Lett., 2008, 8, 2082.

51 R. D. Deegan, O. Bakajin, T. F. Dupont, G. Huber, S. R. Nagel and T. A. Witten, Nature, 1997, 389, 827.

52 T. Hyeon, S. S. Lee, J. Park, Y. Chung and H. B. Na, J. Am. Chem. Soc., 2001, 123, 12798.

53 E. Wetterskog, C.-W. Tai, J. Grins, L. Bergström and G. Salazar-Alvarez, ACS Nano, 2013, 7, 7132.

54 I. Horcas, R. Fernández, J. M. Gómez-Rodríguez, J. Colchero, J. Gómez-Herrero and A. M. Baro, Rev. Sci. Instrum., 2007, 78, 13705.

55 B. Faure, G. Salazar-Alvarez and L. Bergström, Langmuir, 2011, 27, 8659.

56 S. Disch, unpublished work.

57 K. J. Beverley, J. H. Clint and P. D. I. Fletcher, Phys. Chem. Chem. Phys., 2000, 2, 4173.

58 W. Ristenpart, P. Kim, C. Domingues, J. Wan and H. Stone, Phys. Rev. Lett., 2007, 99, 234502.
59 X. Shen, C.-M. Ho and T.-S. Wong, J. Phys. Chem. B, 2010, $114,5269$.

60 P. J. Yunker, T. Still, M. A. Lohr and A. G. Yodh, Nature, 2011, 476, 308.

61 A. F. Routh, Rep. Prog. Phys., 2013, 76, 46603.

62 L. Barash, T. Bigioni, V. Vinokur and L. Shchur, Phys. Rev. E: Stat., Nonlinear, Soft Matter Phys., 2009, 79, 046301.

63 R. Bhardwaj, X. Fang, P. Somasundaran and D. Attinger, Langmuir, 2010, 26, 7833.

64 H. Hu and R. G. Larson, J. Phys. Chem. B, 2006, 110, 7090.

65 A. Stannard, J. Phys.: Condens. Matter, 2011, 23, 83001.

66 J. R. Trantum, Z. E. Eagleton, C. A. Patil, J. M. Tucker-Schwartz, M. L. Baglia, M. C. Skala and F. R. Haselton, Langmuir, 2013, $29,6221$.

67 A. Trybala, A. Okoye, S. Semenov, H. Agogo, R. G. Rubio, F. Ortega and V. M. Starov, J. Colloid Interface Sci., 2013, 403, 49.

68 I. Vancea, U. Thiele, E. Pauliac-Vaujour, A. Stannard, C. Martin, M. Blunt and P. Moriarty, Phys. Rev. E: Stat., Nonlinear, Soft Matter Phys., 2008, 78, 041601.

69 E. Pauliac-Vaujour, A. Stannard, C. Martin, M. Blunt, I. Notingher, P. Moriarty, I. Vancea and U. Thiele, Phys. Rev. Lett., 2008, 100.

70 B. M. Weon and J. H. Je, Phys. Rev. E: Stat., Nonlinear, Soft Matter Phys., 2013, 87. 